\title{
APROXIMACIÓN AL DESEMPEÑO OPERATIVO DE UN MODELO DE REDES NEURONALES EN EL DIAGNÓSTICO DE LA INFERTILIDAD MASCULINA
}

\section{Approach to operational performance of a neuronal network model in the diagnosis of male infertility}

Esteban Velilla-Hernández, Ing, MSc ${ }^{1}$; Paula A. Velilla-Hernández, Bact, MSc, PhD²; Walter Cardona-Maya, Bact, MSc, PhD $^{3}$

Recibido: enero 17/13 - Aceptado: septiembre 5/13

\section{RESUMEN}

Objetivo: realizar una aproximación a la capacidad de las redes neuronales supervisadas para clasificar adecuadamente los hombres fértiles e infértiles utilizando los parámetros seminales convencionales. Materiales y métodos: estudio de corte transversal ensamblado sobre la base de datos del Grupo Reproducción de la Universidad de Antioquia, en el que se escogieron hombres con problemas reproductivos en los doce meses previos y hombres con antecedente de haber tenido hijos. Muestreo por conveniencia. Se tomaron en cuenta: la edad, el tiempo de abstinencia sexual, el volumen del eyaculado, el $\mathrm{pH}$, los porcentajes de movilidad y de viabilidad, y la concentración espermática. Mediante una red neuronal supervisada se desarrolló un modelo de entrenamiento y un modelo de validación.

1 Grupo de Manejo Eficiente de la Energía (GIMEL), Facultad de Ingeniería, Universidad de Antioquia, Medellín, Colombia.

2 Grupo de Inmunovirología, Facultad de Medicina, Universidad de Antioquia, Medellín, Colombia.

3 Grupo Reproducción, Facultad de Medicina, Universidad de Antioquia, Medellín, Colombia. wdcmaya@medicina.udea.edu.co
Resultados: se incluyeron 204 hombres. Para el modelo de entrenamiento 129 hombres, 35 para la validación, 40 para probar el modelo y 25 para la validación externa. En el modelo de la red neuronal clasificó adecuadamente el 90\% de los sujetos con problemas reproductivos y el $91 \%$ de los sujetos fértiles. En el modelo de validación clasificó adecuadamente el $40 \%$ de los sujetos con problemas reproductivos y el 100\% de los sujetos fértiles.

Conclusión: las redes neuronales surgen como una tecnología que podría ser valiosa para el estudio de la infertilidad masculina. Se requieren evaluaciones más rigurosas para definir su real utilidad en el estudio de la pareja infértil

Palabras clave: red neuronal no supervisada, espermatozoide, fertilidad, Colombia.

\section{ABSTRACT}

Objective: To determine the ability of supervised neuronal networks at making the appropriate classification of fertile and infertile men using conventional seminal parameters.

Materials and methods: Cross-sectional study assembled on the database of the Universidad de 
Antioquia Reproduction Group, with a selection of men experiencing reproductive problems within the previous 12 months, and men with a history of having had children. Convenience sampling. The data considered were age, time of sexual abstinence, ejaculate volume, $\mathrm{pH}$, percentage of sperm motility, viability and concentration. Using a supervised neuronal network, a training model and a validation model were created.

Results: Overall, 204 men were included, 129 for the training model, 35 for validation, 40 for testing the model and 25 for external validation. The neuronal network model made the correct classification of $90 \%$ of the subjects with reproductive problems, and 91\% of the fertile subjects. In the validation model, the neuronal network made the correct classification of $40 \%$ of the subjects with reproductive problems, and $100 \%$ of the fertile subjects.

Conclusion: Neuronal networks emerge as a technology that may prove to be valuable for the study of male infertility. More rigorous evaluations are required in order to determine their true usefulness in the study of infertile couples.

Key words: Non-supervised neuronal network, spermatozoon, fertility, Colombia.

\section{INTRODUCCIÓN}

La infertilidad es un problema que afecta alrededor de una de cada seis parejas en edad reproductiva en el mundo, de las cuales aproximadamente un $50 \%$ puede deberse al factor masculino (1). Por este motivo, la evaluación seminal ha sido considerada como uno de los análisis más importantes en el estudio de la pareja infértil. La Organización Mundial de la Salud (OMS) ha reportado valores de referencia para los parámetros seminales, algunos de los cuales han cambiado a través del tiempo (2). Sin embargo, la OMS ha intentado unificar los parámetros de evaluación y sobre todo la metodología para la realización de la evaluación de los parámetros seminales con la publicación periódica de sus manuales para el análisis seminal (2-4).
Las poblaciones involucradas en la evaluación de los parámetros seminales de referencia son muy variables, algunos autores evalúan los parámetros en parejas infértiles (5), otros comparan parejas fértiles e infértiles (6), parejas fértiles (7) y otros han seguido las parejas por años para ver la evolución de la fertilidad (8). En el Grupo Reproducción de la Universidad de Antioquia se han evaluado tanto individuos con fertilidad probada (9-11) como individuos con infertilidad (12-14).

Los resultados obtenidos en las diferentes poblaciones evaluadas no permiten realizar un diagnóstico confiable basado en los parámetros seminales. En un estudio realizado por el Grupo Reproducción se observó que el 54,8\% de un total de 113 hombres con fertilidad probada presentaban al menos un parámetro seminal convencional alterado (9), lo cual evidencia la variación en estos parámetros y la dificultad para realizar un diagnóstico más cercano a la realidad basado en estos resultados.

Las redes neuronales artificiales (RNA) son modelos matemáticos que tratan de imitar las habilidades del cerebro humano ofreciendo ventajas en cuanto a su capacidad de aprendizaje, generalizando situaciones a partir de casos de estudio, funcionamiento en paralelo y tolerancia a fallos por información imprecisa (15). Los modelos de RNA son preferiblemente utilizados cuando no se tiene un conocimiento previo del comportamiento de las variables analizadas, pero existen mediciones, observaciones, registros y datos. Las redes neuronales han sido usadas en diferentes áreas biológicas como la nefrología (16), la microbiología (17), la radiología (18), la neurología (19) y la sepsis (20).

Debido a la carencia de herramientas que permitan diferenciar entre individuos fértiles e infértiles el objetivo del presente trabajo es realizar una aproximación al evento reproductivo desde el lado masculino usando una red neuronal supervisada que permita categorizar la fertilidad de un hombre a partir de los siguientes parámetros seminales: volumen de eyaculado, pH, concentración, movilidad 
I y II, viabilidad espermática, además de la edad del individuo y el periodo de abstinencia sexual previo a la recolección de la muestra de semen.

\section{MATERIALES Y MÉTODOS}

Estudio corte transversal que evalúa los parámetros seminales a partir de los registros de muestras de semen de hombres contenidos en la base de datos del laboratorio del Grupo Reproducción de la Universidad de Antioquia, Medellín, Colombia. Se consideró como hombre fértil a aquel que tenía al menos un hijo o la pareja en embarazo en el momento de la toma de la muestra de semen. Se definió como hombre con problemas reproductivos a los sujetos que presentaban un tiempo superior a 12 meses buscando un embarazo sin resultados exitosos en el momento de la realización del espermograma.

Parámetros evaluados. En cada individuo incluido se evaluó: edad en años, periodo de abstinencia sexual en días, volumen del eyaculado en $\mathrm{mL}, \mathrm{pH}$, porcentaje de movilidad I y II, porcentaje de viabilidad y concentración espermática en millones por $\mathrm{mL}$. Se siguieron las normas establecidas previamente por la OMS (3, 4). La concentración espermática fue evaluada usando la cámara de Makler (21). Como variable resultado se tomó en cuenta ser fértil o no. Se codificó de forma binaria, por tanto, un individuo tiene un valor de fertilidad igual a 1 si tiene fertilidad probada, y un valor de 0 en el caso que presente incapacidad de embarazar a la pareja.

Análisis - Red neuronal artificial supervisada. Una red neuronal está conformada por varios elementos denominados neuronas, los cuales se encargan de procesar la información de entrada con el fin de producir un estímulo o salida (mapeo no lineal entre las entradas y salidas). Una representación matemática de la neurona propuesta por McCullogh-Pitts es la presentada en la ecuación (a), en la cual $X_{i}$ son las entradas, $F T$ es una función de activación o función de transferencia, $W_{i}$ son los pesos asociados a cada conexión creada, $b$ es el offset o bias y $Y$ es la salida de la red.

$$
Y(a)=F T\left(\sum_{i=1}^{n} X_{i} \cdot W_{i}+b\right.
$$

Normalmente una sola neurona no es suficiente para reproducir la respuesta deseada, por lo que se recurre a estructuras o arquitecturas de RNA, siendo la llamada de propagación hacia delante (feed-forward network) la más utilizada en diversas aplicaciones $(22,23)$.

La estructura de la RNA queda definida cuando se establece el número de capas, las funciones de activación de las neuronas de cada capa y los pesos que conectan las neuronas $\left(W_{\mathrm{i}}\right)$. Los parámetros mencionados se deben ajustar con el fin de obtener la respuesta deseada (Target), el ajuste de estos parámetros se logra a través del entrenamiento de la red, proceso en el cual la RNA aprende de la información suministrada; en este proceso se minimiza el error entre la salida de la RNA $\left(y_{i}\right)$ y el valor deseado $\left(t_{i}\right)$. Una métrica que permite observar el ajuste de estos parámetros es el error medio cuadrático dado por la expresión (b).

$$
E_{m s e}=\frac{1}{N} \sum_{i=1}^{N}\left(t_{i}-y_{i}\right)^{2}
$$

En donde $N$ es el número de datos evaluados.

Un método para realizar la optimización de los parámetros y minimizar el error de la expresión (2) es el de Levenberg-Marquardt back propagation, el cual para encontrar los nuevos parámetros (pesos y bias) utiliza la expresión (c).

$$
\chi_{k+1}=\chi_{k}-\left[J^{T}\left(\chi_{k}\right) \cdot J\left(\chi_{k}\right)+\mu_{k} \cdot I\right]^{-1} \cdot J^{T}\left(\chi_{k}\right) \cdot v\left(\chi_{k}\right)
$$

En donde $J$ es el jacobiano que depende del tamaño de la RNA, I es la matriz identidad, $\mu_{\mathrm{k}}$ es la tasa de aprendizaje. 
Modelo seleccionado de red neuronal artificial supervisada. El modelo seleccionado de RNA fue una doble capa hacia adelante con funciones de activación tangente hiperbólica sigmoidea en ambas capas como se presenta en la figura 1. Para el entrenamiento fue seleccionado el método de propagación hacia atrás (backpropagation) (15). Este tipo de estructuras de RNA ha sido generalmente utilizado para el reconocimiento de patrones o en problemas de clasificación, en los cuales para un conjunto de entradas determinadas (inputs) existen unas salidas establecidas (targets), siendo de esta manera una RNA supervisada.

Para la obtención y evaluación del modelo de RNA, los datos de 229 hombres fueron divididos en cuatro grupos: i) 129 datos para el entrenamiento (training), y con este conjunto de datos se ajustaron los pesos y los sesgos de la RNA; ii) 35 datos para la validación, a través de la cual se midió la capacidad de generalización y aprendizaje del modelo encontrado en el entrenamiento; iii) 40 datos para probar el modelo, estos resultados no tuvieron efecto en el ajuste del modelo y se evaluaron una vez este estuvo ajustado (test), y iv) 25 datos para probar externamente el modelo. Los primeros tres grupos de datos -entrenamiento, validación y prueba- fueron seleccionados de forma aleatoria de la base de datos.

Se compara la capacidad del modelo generado por la RNA para clasificar adecuadamente los sujetos con la condición (sensibilidad) y la capacidad del modelo para clasificar de manera apropiada los sujetos sin la condición (especificidad) (24).

\section{RESULTADOS}

Dado que la variable de salida fue la fertilidad y la función de activación de la segunda capa (capa de salida) resultó ser una función tangente hiperbólica sigmoidea, la salida del modelo de la RNA tiene un valor que está en el intervalo entre 0 y 1 incluyendo

Figura 1.

Estructura de la red neuronal artificial

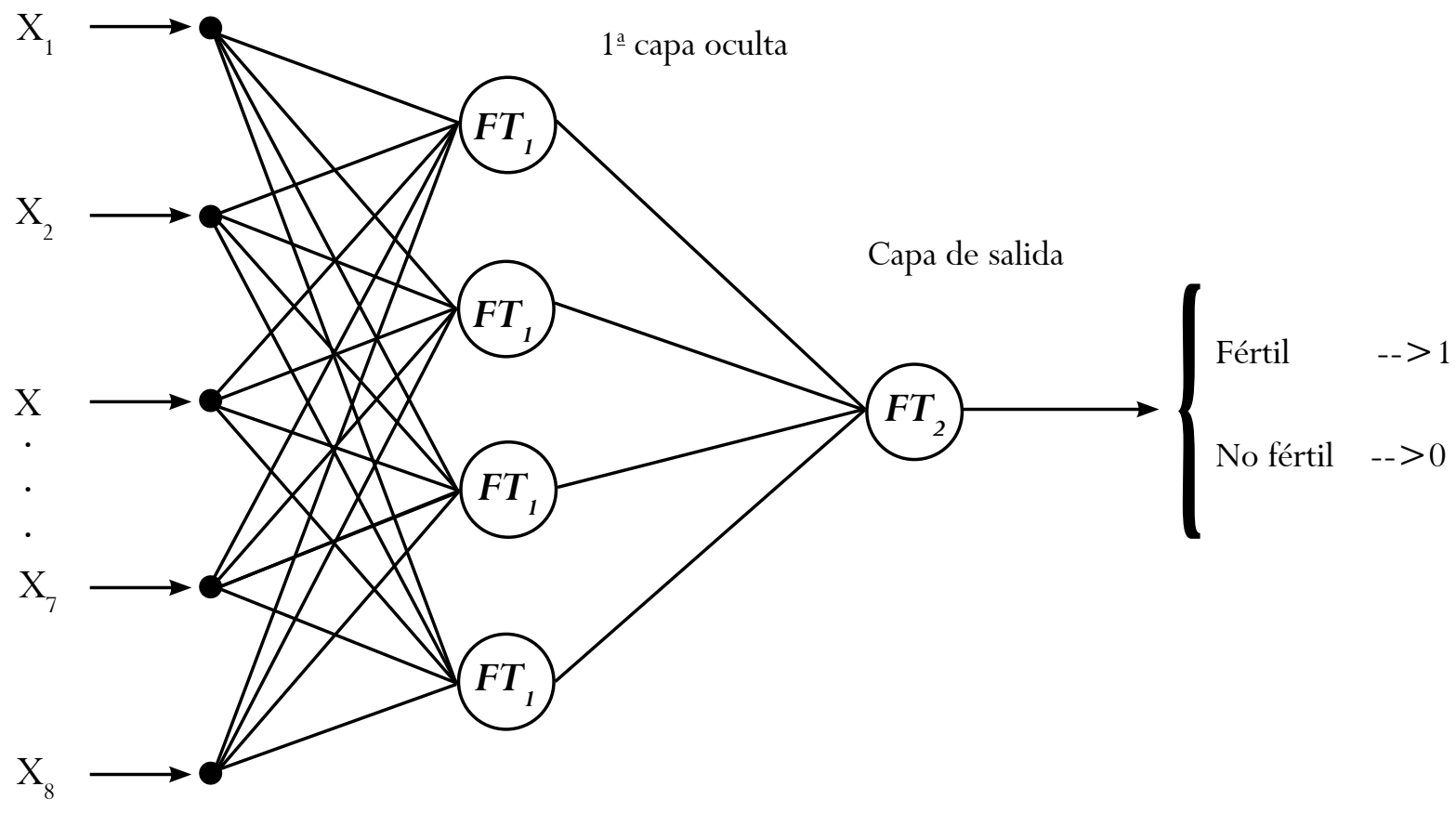

Nodos de entrada 
el 0 y el 1 (figura 2), sin ser exclusivamente 0 o 1 como se hizo en la clasificación de la base de datos.

Se consideraron 51 individuos de la base de datos para probar que la red no estuviera memorizando (cross validation), y 35 individuos para probar el modelo completo, estos 86 datos no fueron considerados en el entrenamiento.

La evaluación inicial de la fertilidad de 118 individuos con la RNA no se concentró en su totalidad en valores de 0 o 1 , sino que están dispersos alrededor de estos, y el valor de 0,5 divide de forma razonable las concentraciones de los puntos (figura 2).

Por tanto, se asumió que los valores iguales o superiores a 0,5 de la evaluación de la fertilidad de los individuos con la RNA serán clasificados como fértiles y los que estaban por debajo de 0,5 serán clasificados como infértiles. De esta manera, la RNA clasificó adecuadamente 90 de los 100 individuos catalogados con problemas reproductivos (sensibilidad 90\%) y 95 de los 104 individuos calificados como fértiles (especificidad: 91,3\%).
En la validación del modelo con el grupo control se encontró que el RNA clasificó como infértiles 6 de los 15 sujetos considerados con problemas de fertilidad (sensibilidad 40\%) y 10 de los 10 sujetos considerados como fértiles (especificidad 100\%).

\section{DISCUSIÓN}

En este trabajo se encontró que las redes neuronales clasificaron adecuadamente entre el 40 y el $90 \%$ de los hombres con problemas de fertilidad y entre el 91 y el 100\% de los hombres fértiles. Los resultados arrojados al usar la RNA indican que la fertilidad no debería ser excluyente como se hizo en la base de datos -es o no es, blanco o negro- (25), sino que esta podría ser evaluada a partir de ciertos grados de fertilidad -unos individuos más fértiles y otros menos fértiles-.

Las redes neuronales ya han sido evaluadas en estudios de fertilidad usando como criterios la penetración de espermatozoides humanos al moco cervical y a los oocitos de hámster dorado (26), o

Figura 2.

Evaluación de la fertilidad utilizando la red neuronal artificial

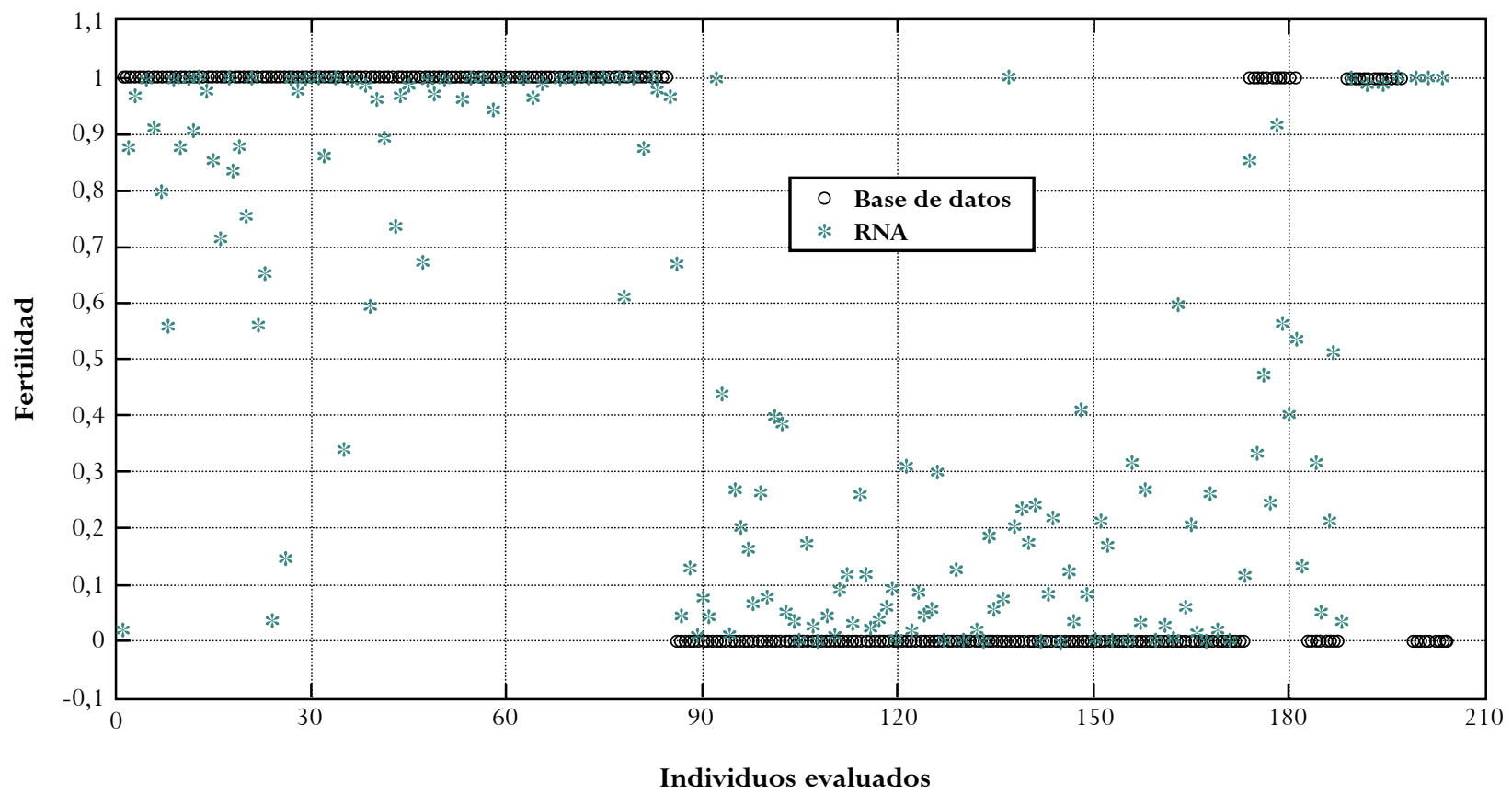


como aproximaciones iniciales para evaluar la morfología espermática en cerdos (27) y humanos (28). Recientemente fue publicado un estudio usando redes neuronales que permite clasificar la calidad de los oocitos en los programas de reproducción asistida, utilizando imágenes de cada uno de ellos y así poder decidir cuáles tienen mejor calidad para ser transferidos (29).

Por tanto, la metodología de redes neuronales supervisadas propuesta en este estudio surge como un método que podría ser considerado para el análisis de los parámetros seminales a fin de clasificar los hombres fértiles e infértiles. En el futuro cercano realizar aproximaciones como la planteada en este trabajo con pruebas seminales funcionales permitirá fortalecer estas aproximaciones y ayudar a la toma de decisiones médicas en el tratamiento de la fertilidad de la pareja, como fue sugerido hace tres décadas por los profesores D. J. Lamb y C. S. Niederberger (30).

Debilidades y fortalezas del estudio. Como limitaciones del estudio tenemos que la definición de hombre con problemas reproductivos tiene limitaciones como proxi de infertilidad masculina lo que afecta el patrón de comparación contra el cual se clasificó el desempeño de la red neuronal. Por otra parte, no es claro que conocer el resultado de la fertilidad de antemano afecte la evaluación del desempeño de la prueba realizada por un sistema informático de red neuronal de la misma manera que la afectaría el juicio de una segunda prueba por un sujeto. Por último, el método permite que haya independencia entre las dos pruebas.

\section{CONCLUSIÓN}

El sistema propuesto en este estudio permitiría ayudar a tomar decisiones médicas sobre la clasificación de un hombre que tiene los resultados de un análisis seminal convencional, con el fin de ayudar en el manejo de los hombres con problemas de fertilidad. Se requieren evaluaciones más rigurosas para determinar la real utilidad de esta tecnología en el estudio de la pareja infértil.

\section{AGRADECIMIENTOS}

Estrategia de Sostenibilidad 2011-2012, Universidad de Antioquia, Grupo Inmunovirología.

\section{REFERENCIAS}

1. Irvine DS. Epidemiology and aetiology of male infertility. Hum Reprod. 1998;13 Suppl 1:33-44.

2. Cardona W. Manual de procesamiento de semen humano de la Organizacion Mundial de la Salud-2010. Actas Urol Esp. 2010;34:577-8.

3. WHO. WHO Laboratory Manual for the Examination of Human Semen and Sperm-Cervical Mucus Interaction: WHO; 1999.

4. WHO. WHO laboratory manual for the examination and processing of human semen: World Health Organization; 2010.

5. Dunphy BC, Kay R, Barratt CL, Cooke ID. Is routine examination of the male partner of any prognostic value in the routine assessment of couples who complain of involuntary infertility? Fertil Steril. 1989;52:454-6.

6. Polansky FF, Lamb EJ. Do the results of semen analysis predict future fertility? A survival analysis study. Fertility and sterility. 1988;49:1059-65.

7. Cooper TG, Noonan E, von Eckardstein S, Auger J, Baker HW, Behre HM, et al. World Health Organization reference values for human semen characteristics. Hum Reprod Update. 2010;16:231-45.

8. David G, Jouannet P, Martin-Boyce A, Spira A, Schwartz D. Sperm counts in fertile and infertile men. Fertil Steril. 1979;31:453-5.

9. De los Rios J, Cardona WD, Berdugo JA, Correa C, Arenas A, Olivera-Angel M, et al. Los valores espermáticos de 113 individuos con fertilidad reciente no mostraron correlación con los parámetros establecidos por la OMS. Arch Esp Urol. 2004;57:147-52.

10. Berdugo J, Andrade-Rocha F, Cardona-Maya W. Parámetros seminales en hombre fértiles de dos poblaciones suramericanas. Arch Esp Urol. 2009;62:646-50.

11. Cardona Maya WD, Berdugo Gutierrez JA, de los Rios J, Cadavid Jaramillo AP. Functional evaluation of sperm in Colombian fertile men. Arch Esp Urol. 2007;60:827-31. 
12. Gil-Villa AM, Cardona-Maya W, Agarwal A, Sharma R, Cadavid A. Role of male factor in early recurrent embryo loss: do antioxidants have any effect? Fertil Steril. 2009;92:565-71.

13. Gil-Villa AM, Cardona-Maya W, Agarwal A, Sharma R, Cadavid A. Assessment of sperm factors possibly involved in early recurrent pregnancy loss. Fertil Steril. 2010;94:1465-72.

14. Rodríguez E, Gil-Villa AM, Aguirre-Acevedo DC, Cardona-Maya W, Cadavid AP. Evaluación de parámetros seminales no convencionales en individuos cuyas parejas presentan muerte embrionaria temprana recurrente: en busca de un valor de referencia. Biomedica. 2011;31:100-7.

15. Haykin S, Network N. A comprehensive foundation. Neural Networks. 2004;2.

16. Gabutti L, Burnier M, Mombelli G, MAL F, Pellegrini L, Marone C. Usefulness of artificial neural networks to predict follow-up dietary protein intake in hemodialysis patients. Kidney international. 2004;66:399-407.

17. Maiellaro P, Cozzolongo R, Marino P. Artificial neural networks for the prediction of response to interferon plus ribavirin treatment in patients with chronic hepatitis C. Current pharmaceutical design. 2004;10:2101-9.

18. Lim WK, Er MJ. Classification of mammographic masses using generalized dynamic fuzzy neural networks. Medical physics. 2004;31:1288.

19. Loukas C, Brown P. Online prediction of self-paced hand-movements from subthalamic activity using neural networks in Parkinson's disease. Journal of neuroscience methods. 2004;137:193-205.

20. Jaimes F, Farbiarz J, Alvarez D, Martínez C. Comparison between logistic regression and neural networks to predict death in patients with suspected sepsis in the emergency room. Critical Care. 2005;9:R150.
21. Cardona-Maya W, Berdugo J, Cadavid A. Comparación de la concentración espermática usando la cámara de Makler y la cámara de Neubauer. Actas Urol Esp. 2008;32:443-5.

22. Meireles MRG, Almeida PEM, Simões MG. A comprehensive review for industrial applicability of artificial neural networks. Industrial Electronics, IEEE Transactions on. 2003;50:585-601.

23. Velilla E, Villada F, Echeverría F. Modelos de pérdida de masa de acero por corrosión atmosférica en Colombia usando inteligencia computacional. Revista Facultad de Ingeniería Universidad de Antioquia. 2009;81-8.

24 Gaitán-Duarte H, Rubio-Romero J, Gómez-Chantraine M. Interpretación del desempeño operativo de las pruebas de tamizaje y de diagnóstico de enfermedades en obstetricia y ginecología. Rev Colomb Obstet Ginecol. 2009;60:365-76.

25. Niederberger C. Computational tools for the modern andrologist. J Androl. 1996;17:462.

26. Niederberger C, Lipshultz L, Lamb D. A neural network to analyze fertility data. Fertil Steril. 1993; 60:324-30.

27. Alegre Gutiérrez E, Sánchez González L, Alaiz Rodríguez R, Domínguez-Fernández JC. Utilización de momentos estadísticos y redes neuronales en la clasificación de cabezas de espermatozoides de verraco. XXV Jornada de Automática Ciudad Real. 2004;2:2.

28. Linneberg C, Salamon P, Svarer C, Hansen LK, Meyrowitsch J, editors. Towards semen quality assessment using neural networks. Neural Networks for Signal Processing 1994. IV Proceedings of the 1994 IEEE Workshop; 1994.

29. Manna C, Nanni L, Lumini A, Pappalardo S. Artificial intelligence techniques for embryo and oocyte classification. Reprod Biomed Online. 2013;26:42-9.

30. Lamb DJ, Niederberger CS. Artificial intelligence in medicine and male infertility. World J Urol. 1993;11:129-36. 